Cinémas

Revue d'études cinématographiques

Journal of Film Studies

\title{
Corps mortels. L'évolution du personnage de Ripley dans la trilogie Alien
}

\section{Sylvestre Meininger}

Volume 7, numéro 1-2, automne 1996

La Représentation du corps au cinéma

URI : https://id.erudit.org/iderudit/1000936ar

DOI : https://doi.org/10.7202/1000936ar

Aller au sommaire du numéro

Éditeur(s)

Cinémas

ISSN

1181-6945 (imprimé)

1705-6500 (numérique)

Découvrir la revue

Citer cet article

Meininger, S. (1996). Corps mortels. L'évolution du personnage de Ripley dans la trilogie Alien. Cinémas, 7(1-2), 121-150. https://doi.org/10.7202/1000936ar
Résumé de l'article

Alien (R. Scott, 1978) est testé aussi célèbre pour son monstre terrifiant que pour son personnage principal, Ripley. Première femme forte du cinéma fantastique mainstream, ce personnage a subi une évolution profonde au cours des deux épisodes suivants, Aliens (]. Cameron, 1986) et Aliens 3 (D. Fincher, 1992). Fait quasi unique à Hollywood, la trilogie se conclut pat sa mort. En constante hésitation quant au degré de proximité entre féminité et monstruosité, la trilogie place le corps de l'héroïne comme enjeu ultime de ses tensions narratives. Ce travail cherchera donc à comprendre l'interaction entre les représentations du monstrueux créées par ces trois films et le personnage interprété par Sigourney Weaver. Nous pourrons alors commencer à comprendre quelles réponses sont ainsi données à la question qui semble hanter Hollywood depuis toujours : «Que faire des femmes? » 


\section{Corps mortels. L'évolution du personnage de Ripley dans la trilogie Alien}

\section{Sylvestre Meininger}

\section{RÉSUMÉ}

Alien (R. Scott, 1978) est resté aussi célèbre pour son monstre terrifiant que pour son personnage principal, Ripley. Première femme forte du cinéma fantastique mainstream, ce personnage a subi une évolution profonde au cours des deux épisodes suivants, Aliens (J. Cameron, 1986) et Aliens 3 (D. Fincher, 1992). Fait quasi unique à Hollywood, la trilogie se conclut par sa mort. En constante hésitation quant au degré de proximité entre féminité et monstruosité, la trilogie place le corps de l'héroïne comme enjeu ultime de ses tensions narratives. Ce travail cherchera donc à comprendre l'interaction entre les représentations du monstrueux créées par ces trois films et le personnage interprété par Sigourney Weaver. Nous pourrons alors commencer à comprendre quelles réponses sont ainsi données à la question qui semble hanter Hollywood depuis toujours: "Que faire des femmes?"

\section{ABSTRACT}

Alien (R. Scott, 1978) has been known as much for its terrifying monster as for its main character, Ripley. The first strong woman in mainstream fantastic cinema, this character underwent a profound change in the course of the two sequels, Aliens (J. Cameron, 1986), and Aliens 3 (D. Fincher, 1992). Almost unprecedented in Hollywood, the trilogy ends with her death. Constantly hesitating over the degree of proximity between feminity and monstrosity, this trilogy makes the 
body of the heroine the site of its narrative tensions. This study seeks to understand the interactions in these films between the representations of the monstrous and the character played by Sigourney Weaver. We can then begin to understand what answer they offer to a question that has long haunted Hollywood: "How to deal with women?"

Le succès important des deux premiers volets de la trilogie Alien laissait présager un excellent accueil pour le dernier épisode. Mais malgré une première semaine exceptionnelle, Alien 3 restera un demi-échec commercial. Difficile de blâmer la promotion qui fut, à l'échelle des moyens engagés dans la production, énorme. Inutile aussi d'accuser David Fincher: la réussite de Seven (1996), tourné de façon très semblable, prouve que le réalisateur n'est pas le facteur décisif dans l'échec de la mécanique hollywoodienne.

La forme même de cet échec peut donner une idée de ses véritables causes. Le public s'eșt d'abord précipité vers ce qui devait logiquement être la conclusion d'une série extrêmement populaire. Or, Alien 3 n'est pas un film raté. Le spectateur y retrouve l'univers mis en place par les deux premiers épisodes, caractéristique importante pour ce type de sequel. On ne s'ennuie pas, l'action rebondit assez efficacement et, même si l'on a peu de temps pour se familiariser avec eux, les personnages sont raisonnablement bien construits et interprétés. C'est manifestement autre chose qui a déplu, compromettant le succès du film en provoquant un mauvais effet de bouche à oreille.

À la fin d'Alien 3, Ripley se laisse volontairement tomber dans les flammes. Cette dernière séquence, culmination dramatique qui donne une dimension assez émouvante au personnage, sonne juste elle aussi. De plus, elle rappelle irrésistiblement la fin de Terminator 2 (J. Cameron, 1992), qui fut une très grande réussite commerciale. Où se situe donc le problème? Pourrait-il se rapporter au fait que Ripley, contrairement au cyborg, soit une femme?

En tant que troisième épisode, Alien 3 devait apporter certaines réponses aux questions soulevées par les deux premiers. Pour comprendre en quoi ce film a déçu, il sera donc nécessaire d'exa- 


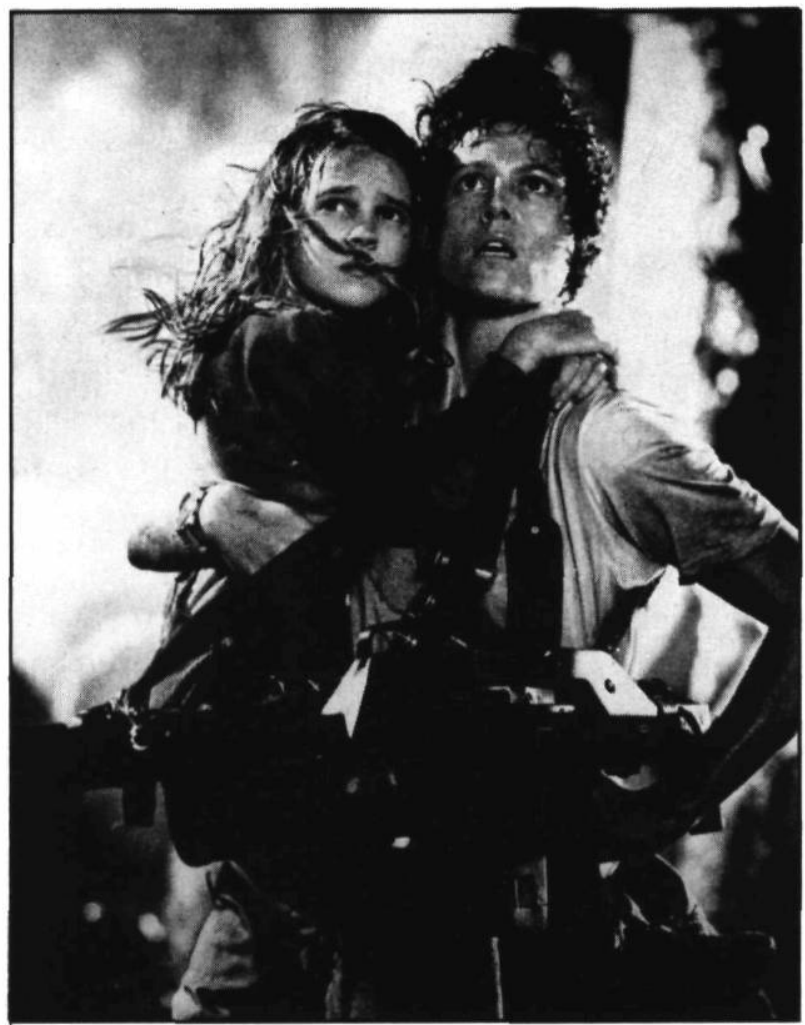

\section{Alien de Ridley Scott (1978)}

Collection Cinémathèque québécoise

miner ses deux prédécesseurs en essayant de déterminer la nature et la portée de ces questions exigeant réponses. Ce sont en effet ces interrogations, et les angoisses ou les désirs qui les animent, qui désignent le plus clairement les enjeux de la trilogie en tant que produit de la culture états-unienne contemporaine.

Mon analyse aura plusieurs bases théoriques visant à cerner ces enjeux en envisageant les textes de manières complémentaires. Je m'appuierai, sur un plan purement psychanalytique, sur les recherches de Melanie Klein et Margareth Mahler. Mes observations se baseront sur leur conclusion la plus importante: la mère est l'objet dans lequel sont placés les sentiments les plus 
contradictoires de l'enfant, sentiments qui perdurent tout au long de notre vie adulte.

Au-delà de la psychanalyse, j'utiliserai aussi les travaux de Julia Kristeva et de Klaus Theweleit ' qui définissent, à travers l'analyse de textes très différents, le rapport occidental au corps.

\section{Le ventre sans fin: "In space, no one can hear you scream"}

La réussite d'Alien tient il est vrai à un scénario impeccable, mais peut-être plus encore à la brutalité de ses représentations. Les images qui habitent le film frappent violemment parce qu'elles oscillent assez subtilement entre l'évocation et la monstration pure et simple, une mince épaisseur de narration nous empêchant à peine de prendre immédiatement conscience de ce que nous voyons.

L'épave où les explorateurs pénètrent en est la plus vivace représentation. Lieu fondamentalement inconnu par son origine même, sa forme est, elle, immédiatement reconnaissable. Deux immenses trompes de Fallope, ou deux jambes écartées, se rejoignent en un utérus aux trois quarts enfoui sous la surface et, à l'abri dans cette immense matrice, des œufs attendent un contact.

À l'intérieur, tout est sombre, humide, distordu. Les couloirs sont étroits, étouffants, les salles immenses et sans fins, leurs parois tapissées de reliefs rappelant des ossements humains.

La Terreur prend ici sa source dans la représentation d'un immense corps inconnaissable qui évoque un souvenir partagé par tout le public. De façon évidente, et ce pour n'importe quel spectateur, c'est le pouvoir engloutissant de ce lieu qui crée à la fois la fascination et l'angoisse. Il suffit, pour s'en convaincre, de remarquer à quel point le filmage insiste sur cet aspect. Kane est isolé par des plans rapprochés ou noyé dans une noirceur impénétrable qui contribue à le faire disparaître peu à peu. Il est fatigué, coupé de ses compagnons par des interférences et plongé dans un silence total, troublé seulement par sa propre respiration. Notre peur vient très immédiatement du danger d'engloutissement, de disparition que court le personnage. Nous ne savons pas ce qu'il va découvrir, mais surtout nous avons peur de nous perdre avec lui dans cet espace sans limites ni repères. 
Un tel lieu renvoie aux angoisses infantiles liées à la mère définies par les travaux de Margareth Mahler et Melanie Klein. Cette séquence d'Alien fonctionne car elle donne à voir un corps, correspondant à notre vision fantasmatique du corps maternel.

Le cadavre du pilote que les explorateurs retrouvent dans la grande salle centrale précise cette première observation. Cet être fut une victime des monstres, comme en témoigne le trou qui perce sa cage thoracique. Caractérisé comme masculin par l'énorme appareillage phallique qui le domine ${ }^{2}$, cet "homme" s'est lui aussi irrémédiablement perdu dans le vide hostile de ce ventre meurtrier. Malheureusement, le petit groupe ne comprend pas l'avertissement. Kane descend seul par un puits et pénètre dans la salle où sont entreposés les œufs.

Ces œufs font écho au reste du navire par leur forme et leur fonction quasi utérines, et par l'ouverture vaginale située à leur sommet ${ }^{3}$. Lorsque l'homme en touche une, elle s'écarte lentement avec un bruit spongieux pour révéler un intérieur organique repoussant. Il se penche et nous avons juste le temps de voir quelque chose jaillir vers lui. La séquence suivante montre le parasite accroché à son visage. Le contenu de l'œuf est bien en adéquation avec le reste de ce vaste utérus: féminoïde ${ }^{4}$, terrifiant et hostile parce que dévorateur. Insistons sur cette notion d'hostilité : les œufs attendent, animés et dirigés par une "volonté" maléfique.

N'oublions pas une caractéristique importante de l'épave: le mélange entre matières métallique et organique, entre appareillages technologiques et tissus vivants. Ce mélange est caractéristique du film et est présent dans la créature elle-même, comme dans toute l'œuvre de Giger. Ce lieu existe au-delà des frontières rassurantes entre technologie et humanité présentes à bord du Nostromo. À l'intérieur de ce ventre sans fin tout est mêlé, et cette perte de différenciation n'est pas perçue comme positive. Au contraire, elle est conçue pour inspirer le dégoût et la peur. Comme l'explique Kristeva, le corps de la mère est un lieu terrifiant parce que tout s'y mélange, faisant disparaître les différences et les séparations fondatrices de notre identité. Il semble donc que le danger menaçant les protagonistes prenne son origine dans une caricature du corps et des fonctions biologiques 
maternelles. Et nous allons voir que c'est bien la mère qui domine tout le film.

On retrouve la même angoisse dans les couloirs sinistres du Nostromo. Lui aussi composé de corridors et de quartiers exigus, de salles démesurées, il est conçu et filmé pour désorienter le spectateur et créer une sensation d'étouffement. C'est dans ses sombres entrailles que les personnages trouvent la mort aux mains de la créature, et rien n'est conçu pour les aider dans leur lutte. Au contraire, le monstre profite des recoins et conduits innombrables pour échapper à ses poursuivants et les tuer un à un. Comme l'épave, le Nostromo n'est qu'un intérieur sans extérieur, un lieu sans perspective, dont on ne peut avoir une vue d'ensemble. Un lieu qui nous empêche donc de nous positionner comme "je " clairement indépendant de ce qui l'entoure.

Mais le Nostromo, comme l'épave, ne sont que le corps de la Mauvaise Mère. Son esprit s'incarne dans Mother, l'ordinateur de bord chargé de s'occuper de l'équipage. Omnisciente et omnipotente, cette volonté malfaisante parcourt tout le film et participe activement à la mort de ses enfants.

D'abord nourricière, elle semble s'occuper convenablement de l'équipage. Elle lui donne naissance dans la propreté froide mais rassurante de la salle d'hibernation, puis le nourrit ${ }^{5}$. Même si elle n'accomplit pas son travail avec beaucoup de soin, le film insiste sur son lien symbiotique avec l'équipage: elle pilote le Nostromo pendant l'hibernation, avertit l'équipage de la présence d'un signal étranger et lui dicte sa conduite par l'intermédiaire de Dallas, le capitaine. Mais la suite du film révèle sa duplicité. Nous apprendrons en effet qu'elle connaissait les dangers auxquels ils seraient exposés. Lorsque Ripley lui demande de l'aider, l'ordinateur répond froidement que la survie de l'équipage n'a aucune importance. Enfin, Mother refuse d'interrompre la procédure d'autodestruction alors que Ripley est encore à bord. Le comportement de cet esprit maternel est aussi tortueux et dangereux que l'est son corps. Sa psychologie correspond à celle de la Mauvaise Mère qui, à la fois autoritaire et menteuse, abandonne ses enfants à leur sort.

Examinons à présent les deux menaces physiques du film, l'androïde Ash et la créature. Loin de la naïveté qui a longtemps 
présidé à l'élaboration des monstres cinématographiques, cet Autre, comme le requin de Jaws (Steven Spielberg, 1975) et les jouets guerriers de Star Wars (Georges Lucas, 1977), marque l'entrée de l'industrie hollywoodienne dans le règne de la technologie extradiégétique: il demeure encore aujourd'hui extrêmement convaincant. Mais de façon intéressante, le monstre est surtout crédible, et terrifiant, parce qu'on ne le voit pas.

La créature a d'abord surgi d'un ventre maternel difforme et inquiétant. Indissociable de cette origine féminine, elle continue de poser les processus de gestation et de naissance organiques comme repoussants et dangereux, pour l'homme en particulier. Le film insiste sur sa capacité à pénétrer, à envahir l'environnement humain. C'est bien son incapacité à maintenir l'Autre à l'extérieur, du navire ou du corps, qui condamne l'équipage. Mais dès le début, lorsque les explorateurs ramènent leur infortuné compagnon sur le Nostromo, cette invasion se fait par l'intermédiaire du corps masculin. Accrochée par ses pattes griffues au visage de Kane, la larve le viole en introduisant un long appendice dans sa bouche. Même si le spectateur ne connaît pas encore les conséquences de ce contact, son caractère horrifique annonce les dangers qu'il représente.

En émergeant du ventre de Kane, la créature achève la destruction de l'identité masculine en plaçant l'homme dans une position éminemment féminine. Après s'être perdu une première fois au fond de l'épave, puis avoir été envahi dans sa chair, celui-ci est finalement absorbé dans le cycle reproducteur horrifiant de la Mère. Le résultat de cette ultime transgression de nos frontières biologiques et culturelles confirme bien l'incompatibilité des deux genres: leur union ne peut produire que l'horreur.

Ce monstre qui a paru, et paraît souvent encore, si nouveau et si "étranger" aux spectateurs, nous impressionne avec la même force que les décors du film parce qu'il nous atteint aussi directement. Conçu par le même artiste, et surtout animé par les mêmes angoisses, nos angoisses, il s'adresse explicitement à notre inconscient, et ce principalement parce qu'il est invisible, c'est-àdire innommable. Comme l'épave, la créature, à la fois poulpe et insecte, est composée d'un mélange entre matières métallique et organique. Protéiforme et en symbiose avec l'univers obscur du 
Nostromo, elle n'a pas d'identité précise. Elle est ainsi placée entièrement du côté de la Mère archaïque, à l'opposé de notre monde rationnel et soigneusement catégorisé.

Mais là n'est pas la seule frontière qu'elle nie. Plus intéressant encore, son corps regroupe des attributs masculins et féminins, alternativement mis en valeur par le film. Ainsi, lorsqu'elle va tuer Parker, personnage caractérisé comme viril, sa double bouche garnie de dents acérées représente une menace castratrice. Mais lorsque le monstre s'attaque à Lambert, une femme faible paralysée par la terreur, il fait remonter sa queue le long des jambes de sa victime. Même attitude face à Ripley, devant qui il exhibe son crâne et sa bouche phalliques. Cet Autre fait peur parce qu'il n'est jamais le même, en fait parce qu'il n'est jamais un mais plusieurs à la fois. Masculin et féminin, il devient terrifiant parce qu'impensable, incompréhensible dans le cadre de notre perception sexuée de la réalité.

Il semble que l'influence de la Mauvaise Mère efface les différences jusqu'à rendre impossible la distinction sexuelle. Sa progéniture ${ }^{6}$ est, elle, caractérisée par la démesure de ses attributs sexuels (bouches, tête, queue-tentacule). Cette hypersexualité est liée à une puissance destructrice face à laquelle, comme l'explique Ash, les humains ne peuvent rien. Ici, trop hostile rime avec trop organique et, comme nous venons de le voir, trop sexuel. Le monstre est donc dangereux parce qu'il est une émanation de la Mauvaise Mère, et aussi parce qu'il représente une énergie sexuelle incontrôlable. La réussite du film, mesurée d'après sa capacité à créer la peur, se trouve dans cette combinaison.

A priori, Ash semble tout à fait étranger à l'univers sombre et moite de la créature. Froid, rationnel, déterminé, il semble plutôt caricaturer une volonté de contrôle connotant la masculinité. Mais il rejoint la Mauvaise Mère, d'abord narrativement, puisqu'il trahit l'équipage, puis dans sa représentation même, lorsqu'il est décapité. Un liquide visqueux se met alors à jaillir de son cou et nous apercevons la masse informe qui constitue son intérieur. Dès que les limites de son corps sont ainsi percées, Ash change radicalement de comportement. Auparavant tueur calculateur et maître de lui, il n'est plus qu'un corps hystérique, agissant sans discernement, ni mesure. Dans le cadre du film, la 
révélation de sa nature organique ne peut que le rapprocher du monstrueux. Si ce processus semble assez différent de celui décrit pour la créature, il fait pourtant surgir l'horreur de la même manière. Là encore, quelque chose de terrifiant et d'incontrôlable attendait, à l'intérieur, d'être libéré pour détruire le monde rationnel des humains.

\section{Pourquoi Ripley survit-elle?}

Entourés, dominés et absorbés par ces émanations d'une puissance maternelle dévoratrice, les membres de l'équipage périssent tous et seule Ripley parvient à s'enfuir, ce qui nous amène à la dernière séquence du film. Comme le montre Carol J. Clover dans son livre Men, Women and Chainsaws ${ }^{7}$, il est important de noter que ce héros féminin est le résultat évident d'une volonté promotionnelle de la part des producteurs ${ }^{8}$. Il ne faut pas pour autant renoncer à décrypter les multiples fonctions qu'elle va prendre au cours de cette séquence, à savoir objet de notre regard puis sujet actif de l'action.

Une analyse de ce passage se basant sur "Visual Pleasure and Narrative Cinema" de Laura Mulvey placerait ici le spectateur masculin comme voyeur omnipotent, l'attaque sadique du monstre matérialisant cette domination et devenant une source de plaisir visuel. Mais Carol Clover, dès l'introduction de son livre", propose de dépasser cette lecture "caméra = regard masculin ". Au contraire de Mulvey, Clover accepte l'évidence: l'identification du public avec Ripley. Le spectateur, qu'il soit homme ou femme, est alors positionné comme victime directe du monstre et de son agression sur le corps de l'héroïne.

Cette analyse nous semble satisfaisante dans la mesure où elle permet de comprendre la peur et le plaisir ressentis par tous les spectateurs face au film. Un plaisir qui vient des changements de statut narratifs que nous subissons pendant le récit. D'abord victime avec les personnages secondaires ${ }^{10}$, le public goûte une satisfaction de type masochiste, satisfaction renforcée par la certitude que le cours des événements va de toute façon s'inver-

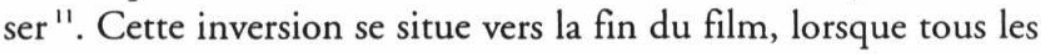
compagnons de la final girl, notamment tous les hommes, ont été tués. 
Il est clair qu'Alien suit à la lettre ce schéma. Mais avant d'affronter son ennemi, Ripley révèle son corps à notre regard. Au milieu des images terrifiantes de la féminité qu'offre le film, le corps à moitié nu de Sigourney Weaver a un effet précis. Ripley se déshabille après être parvenue à s'échapper du Nostromo quelques secondes avant son explosion. C'est un moment de repos, pour elle comme pour le spectateur, et la vision de son corps désirable efface les souvenirs pénibles accumulés au cours du film. Fondamentalement opposé aux formes grotesques et hostiles des multiples incarnations de la féminité prédatrice, ce corps mince et harmonieux est agréable parce que normal (c'està-dire contenu, maîtrisé). Considérer cette séquence comme un simple moment de voyeurisme serait donc ignorer sa position par rapport au reste du film. Placée à la toute fin, elle fonctionne aussi comme un démenti, une négation de tout ce que nous avons vu, une manière de nous rassurer sur la biologie féminine. Le rôle de régulateur culturel du film hollywoodien apparaît donc clairement ici. Là où l'épave n'était qu'une immense ouverture sans fond, Ripley est une, entière, une ravissante enveloppe dissimulant soigneusement son intérieur à notre vue. Là où le monstre bavant et sifflant existait dans l'intermédiaire et l'indéterminé, Ripley reste sagement dans les limites définissant notre identité sexuelle. Voilà aussi pourquoi elle doit survivre.

Puis, lorsqu'elle revêt une épaisse combinaison spatiale et saisit un pistolet à grappin, Ripley devient la final girl de Clover. Elle cesse de se comporter selon les règles de la féminité définies par le film d'horreur ${ }^{12}$ et devient une "femme masculinisée ${ }^{13}$ ". Ce processus, étroitement lié au nouveau rôle narratif de l'héroïne, sera pleinement confirmé par Aliens, comme nous le verrons plus loin ${ }^{14}$.

Alien pose les bases de la série: une représentation cauchemardesque de la sexualité féminine donne naissance à une créature dont l'hostilité dépasse l'entendement. L'utilisation des effets spéciaux est à ce titre remarquable. Le film de Ridley Scott prend le contre-pied esthétique exact de Star Wars, parce qu'il veut parler de ce qui s'y oppose. La célébration romantique d'une guerre entièrement technologisée, où même une épée ne 
fait pas jaillir le sang, demandait une visibilité totale. Ainsi présent, immédiatement accessible, le mouvement grisant des machines et de la guerre promettait la vie éternelle aux hommes qui sauraient le maîtriser. Là où Georges Lucas veut, et parvient, à tout montrer, Scott cache, simplement parce qu'il doit évoquer l'indicible, ce qui fonde notre identité lorsque nous nous en séparons: la mère, dans la perception archaïque que nous en avons.

Il est alors naïf de croire, comme l'ont fait certains critiques, que le film construit l'image d'une féminité "libérée ". Ripley incarne simplement une féminité tolérable parce que contrôlée. L'enjeu culturel principal du film se trouve dans cette constatation. À la question, pour le moins préoccupante, "existe-t-il une féminité acceptable, à laquelle la civilisation puisse survivre?", Alien répond in extremis par l'affirmative. Le second épisode continue sur cette voie, mais en opérant de manière plus tranchée.

\section{Aliens, la réponse rationnelle: "This time it's war"}

Produit à l'apogée des années Reagan et réalisé par James Cameron 's, Aliens cherche à effacer les doutes et les peurs évoquées par son prédécesseur. Pour l'Amérique "renaissante" de cette décennie, l'espace intersidéral ne peut rester un lieu étranger et inquiétant laissé aux mains de puissances féminines indestructibles. Comme les autres, ce territoire doit être pénétré et conquis, et les forces armées sont les mieux placées pour procéder à cette colonisation ${ }^{16}$. Si Alien tirait sa force dramatique d'une représentation d'un Inconnu inconcevable, Aliens veut cerner cet Inconnu, lui donner un visage et une forme pour mieux le voir, et donc le détruire. Pour Alien, le doute, ce qui mettait le spectateur mal à l'aise, venait principalement de l'ambiguïté sexuelle du monstre et de la transgression des genres provoquée par sa naissance, mais aussi d'une fascination esthétique avouée pour l'Autre. Aliens cherche constamment à effacer ces contradictions insupportables en opposant des forces bien distinctes. Bien sûr, nous verrons que l'axe de cette opposition est celui de l'identité sexuelle.

L'excitation ne naît plus ici du doute et de la paranoïa, mais du spectacle fascinant de la guerre, de l'affrontement direct 
entre des soldats et un Autre clairement défini. À ce titre, les analogies entre ce film et ceux décrivant la guerre du Vietnam sont nombreuses. Les soldats quittent un lieu rassurant pour arriver par les airs au milieu d'un environnement hostile. La principale caractéristique de cet environnement est de bloquer la vue et de réduire la liberté de mouvement. L'équipement des marines est perfectionné et est uniquement constitué d'armes pouvant toucher, et donc maintenir, l'ennemi à distance. Mais il ne peut venir à bout de leurs adversaires qui savent se cacher et attaquent en masse sans relâche. Enfin, ils sont trahis par leurs deux supérieurs: économique (Burk, un homme corrompu envoyé par la Compagnie), et militaire (Gorman, un gradé qui ne connaît la guerre que par les livres, et qui sera incapable de faire face au danger).

Alors que le premier épisode condamnait irrémédiablement la Compagnie, Aliens semble la juger moins durement. Le personnage de Burk, stéréotype du yuppie juif, en rassemblant les traits négatifs ${ }^{17}$ de la corporation géante (inhumanité, avidité frénétique et dangereuse), permet de les reporter sur un seul individu, et donc d'innocenter celle-ci sur deux niveaux. En tant que Mère et en tant qu'incarnation du système capitaliste.

\section{Premier axe d'opposition : le féminin comme Autre absolu}

Abandonnant les subtilités du premier épisode, Aliens rentre immédiatement dans le vif du sujet, après une première séquence racontant le sauvetage de Ripley. Celle-ci se réveille d'un cauchemar où elle se voyait donner "naissance" à un monstre. La scène est tournée de façon à être très impressionnante et annonce le thème central du film: la lutte entre deux forces opposées pour la maîtrise du corps féminin.

L'état dans lequel les militaires trouvent la colonie confirme les pires craintes suscitées par le rêve de Ripley. Déjà, nous retrouvons le lieu angoissant d'Alien. Couloirs étroits et salles encombrées limitent la visibilité et enferment les personnages dans un espace étouffant.

Après avoir retrouvé Newt, les marines décident de partir à la recherche des colons et se dirigent vers le réacteur de la station. C'est là qu'ils découvrent l'origine de ces destructions. Envahis 
par les créatures, les sous-sols ont été transformés en boyaux sinueux tapissés d'une résine verdâtre et humide. Conçus pour contraster avec les locaux dépouillés et fonctionnels de l'environnement humain, ces décors matérialisent l'intrusion incontrôlable d'une nature fondamentalement hostile. Pour arranger les choses, Ripley explique que leurs armes pourraient endommager un réacteur nucléaire. Gorman, l'officier supérieur caractérisé comme incompétent, ordonne aux soldats de les décharger avant de pénétrer dans le labyrinthe organique.

$\mathrm{Au}$ bout de ses couloirs inquiétants se trouve une immense salle. Une femme encore vivante les supplie de la tuer. Avant qu'ils ne comprennent, une créature surgit de son torse et ils doivent l'incinérer avant qu'elle ne s'échappe. Regardant autour d'eux, les soldats découvrent les restes des colons enchâssés dans la résine. Tordus, éventrés et grimaçant, ils témoignent du sort qui attend le petit groupe. Alors, sortant littéralement des parois, les monstres se jettent sur les marines et les font disparaître un à un. La confusion est totale et le filmage insiste bien sur l'impossibilité de voir l'adversaire. Gros plans sur des visages paniqués, ombres se déplaçant rapidement, montage trop rapide, hurlements d'horreur: cette menace est inhumaine, invincible parce qu'incompréhensible. Même les lourdes mitrailleuses que deux soldats avaient conservées ne parviennent pas à endiguer ce flot, qui submerge tout. De ce chaos reviennent seulement trois survivants, dont Hicks, un homme nonmachiste, et Vasquez, une chicana très masculine et sûre d'elle.

La première partie du film montre comment une force naturelle a transformé le monde rationnel des hommes en une caricature repoussante du corps féminin, et l'opposition féminin/ masculin est systématiquement reconduite. Tout d'abord, les hommes (et les femmes soldats) sont virils et disciplinés. Mais cette discipline n'a pas fait disparaître leur individualité : les trois plus importants (Hicks, Vasquez, Hudson) sont interprétés très différemment et restent facilement reconnaissables. Plus généralement, tous respectent les ordres mais méprisent ouvertement le respect pointilleux du règlement observé par Gorman, dont le manque de caractère les met en danger. C'est d'ailleurs parce que deux d'entre eux désobéissent en conservant leurs armes que 
quelques-uns échapperont à la mort. L'intégrité du groupe est donc garantie par la camaraderie plutôt que par une obéissance aveugle à la hiérarchie. En cela, il est clair que les humains s'opposent au féminin monstrueux. Pour le spectateur, leur comportement caricaturalement individualiste permet de se raccrocher, en s'y identifiant, à des corps positifs parce que différents, les uns des autres, et de leur environnement.

Ils portent un équipement imposant et perfectionné (fusils, radios, détecteurs, véhicules divers, armures, etc.) aux formes anguleuses, qui produit continuellement des sons métalliques, soulignés à plaisir par la bande-son. Reliés au poste de commandement par des caméras montées sur leurs casques et armés à la dernière mode, ils utilisent une technologie performante qui facilite leur contrôle de la situation, notamment par sa capacité à étendre leur vision. Et c'est lorsque cette technologie commence à devenir inefficace qu'ils se sentent et deviennent vulnérables.

Associées à des sons et des images liquides et spongieux, sans individualité définie, les créatures se confondent entre elles, et avec leur habitat même. Cette symbiose semble leur permettre de surgir de nulle part pour attaquer traîtreusement les soldats, sans que ceux-ci aient le temps d'exercer leur vision, et donc leur puissance de feu, sur elles. Caractéristique importante, elles cherchent continuellement à établir un contact, à toucher pour saisir et emporter. Comme le robot liquide de Terminator 2, elles sont dangereuses parce qu'elles veulent entrer en contact physique avec les hommes.

Poursuivant les humains, elles se cachent, s'infiltrent et cherchent par tous les moyens à pénétrer leur espace. Lorsqu'elles se révèlent pour attaquer, elles se jettent par dizaines sur les marines qui ne résistent que grâce à leur puissant armement. Terminator 2 employait aussi la plupart de ses effets spéciaux à montrer comment sa machine fantastique pouvait échapper à la vue, ou pénétrer de force les défenses érigées par ses victimes.

Face à cette marée organique, l'ordre rigide des militaires va se transformer en panique et leur progression ordonnée en débandade. Virilité et technologie ne sont plus rien à l'intérieur du sombre labyrinthe de la sexualité féminine: l'homme pénètre la femelle à ses risques et périls. 


\section{Seconde opposition : deux femmes, deux mères. Séparer pour purifier}

Après de multiples péripéties, Ripley et Newt se retrouvent seules au fond de la tanière des monstres. Là, mère et fille vont être confrontées à l'origine du mal: la Reine. Croisement d'araignée et de mante religieuse, son corps est entièrement noir et il est d'abord difficile d'en comprendre l'organisation. Devant elle s'étalent des dizaines d'œufs alors qu'il ne reste aucun humain où implanter des larves: sa biologie est totalement hors de contrôle, hors des limites du raisonnable. Comme si elle comprenait la colère toute maternelle de son homologue, Ripley commence à reculer en portant Newt dans ses bras. Mais un œuf s'ouvre brusquement et, paniquée, elle incendie la pièce avec un lanceflammes ${ }^{18}$.

Après une fuite éperdue, Ripley parvient à retourner sur le navire principal, situé en orbite au-dessus de la station. Mais la Reine a réussi à la suivre jusque-là et se jette sur Newt qui parvient à lui échapper momentanément. Ripley attire alors son attention, puis s'enfuit pour revenir à l'intérieur d'un robot monte-charge. La séquence est construite pour montrer clairement que les deux mères s'affrontent pour la possession de Newt, et c'est la bonne mère qui triomphe.

Cette deuxième partie, en opposant constamment deux principes maternels, expose et résoud le problème principal du film. Des deux féminités, la première est consciente, choisie et maîtrisée, l'autre incontrôlable et monstrueuse. Nous apprenons au début du film que la fille de Ripley est morte de vieillesse pendant que sa mère dérivait dans l'espace. Elle est donc seule jusqu'à ce qu'elle découvre Newt. Cette nouvelle famille est complétée par le caporal Hicks, qui se rapproche sensiblement de Ripley avant d'être blessé' ${ }^{\prime}$.

Face à eux se trouve une caricature effrayante du processus reproducteur féminin. Cette représentation est présente aussi bien dans les décors que dans les monstres. La Reine, tapie au fond de son labyrinthe, en est le paradigme. Deux différences principales la séparent du monstre d'Alien. Tout d'abord, elle n'est plus ambivalente sexuellement. Nous avons bien affaire avec une créature dont l'apparence et le comportement connotent la 
féminité. D'autre part, elle se reproduit, et son pouvoir de destruction réside explicitement dans cette capacité.

Si Ripley est dépourvue de toute biologie (elle a adopté Newt), la Reine incarne une puissance reproductrice, indiscutablement féminine, terrifiante, située en dehors des institutions de contrôle de la société et menaçant de tout détruire sur son passage. Son apparence est dictée par des angoisses primitives liées aux organes sexuels de la femme dont l'intensité est telle qu'elle doit être précipitée dans l'espace.

\section{Ceux qui vivent en bas}

Comme un grand nombre de films produits à cette période, Aliens oppose deux femmes ${ }^{20}$. Le nœud du conflit est bien sûr la famille, ici symbolisée par Newt, dont la survie dépend de sa résistance à l'invasion par l'Autre. L'affrontement est certes marqué par de lourdes connotations raciales et de classe. Dans son article "Monstruous Mothers, Medusa, Grendel and Now Aliens", Linda Bundtzen montre que Ripley, Blanche et issue de la classe moyenne, semble bien combattre ici le bouc émissaire favori de l'ère Reagan/Bush: la mère de race noire vivant sur les aides gouvernementales, dont le pouvoir reproducteur met en danger l'économie du pays ${ }^{21}$. Souvent décrite comme un parasite de l'État profitant des impôts payés par les travailleurs états-uniens, cet Autre fantasmé nourrit aussi un personnage tel que la Reine.

Le personnage de Bishop apporte aussi une nuance de classe à celui de Ripley. Celle-ci est d'abord horrifiée d'apprendre qu'un androïde fait partie de l'expédition. Lorsqu'il se montre amical, elle le rejette avec haine et mépris. Mais elle va comprendre qu'il est prêt à sacrifier sa vie pour celle des humains. Ainsi, c'est lui qui viendra la chercher alors que la Reine la poursuit. Il sauvera aussi Newt en la rattrapant avant qu'elle ne soit éjectée dans l'espace à la fin du film. Cet esclave dévoué, incapable de nuire aux humains, se comporte comme le "bon nègre" si souvent présent dans les films états-uniens d'avant-guerre ${ }^{22}$. D'abord inquiétant par son altérité et le lourd passé de ceux de sa race (ici, Ash dans Alien), il se révèle positif parce qu'il sait rester soumis aux humains. Sa qualité d'inférieur assure alors le statut dominant des autres personnages. Bien sûr, cette domination est ici entiè- 
rement justifiée par le fait que Bishop n'ait rien d'humain, mais le plus important est qu'elle montre clairement que les soldats, et Ripley, n'appartiennent pas au prolétariat de ce futur.

\section{Ripley: héros moderne}

J'ai déjà évoqué la volonté du film de rétablir un ordre dangereusement déstabilisé par le premier épisode. Ce rétablissement est d'abord marqué par la reconstitution d'une famille conventionnelle et par la fascination avouée du film pour les armes et les militaires. Mais, plus profondément, questions et angoisses demeurent et c'est autour du corps féminin que la bataille se joue. Le rêve de Ripley a posé les données du problème : à qui va appartenir son corps, de quel côté sa biologie va-t-elle basculer? La féminité est-elle irrémédiablement incontrôlable et dangereuse? Et surtout, comment en venir à bout, comment la circonvenir? Le film répond à ces interrogations en séparant la notion occidentale de féminité en deux personnages antagonistes. La Reine incarnait le monstrueux, mais comment a évolué Ripley?

Aliens reproduit le processus du premier épisode en le poussant plus loin. Apparemment stimulée par les leçons de Hicks sur le maniement des armes, notre héroïne se confectionne un fusil lance-flammes et se charge de grenades et de munitions diverses. Même si nous savions déjà qu'elle était courageuse et capable de se défendre, il s'agit là d'un complet retournement de situation. Se conformant au comportement typique de la final girl, elle s'arme et se masculinise, avant de passer à l'attaque. Mais ici, cette phase est particulièrement extrême: Ripley se transforme en soldat d'élite.

Comme à la fin du premier épisode, nous comprenons une fois encore que Ripley a bien fait de se protéger ainsi. Couloirs sinueux et étouffants font monter la tension par la constante menace d'une promiscuité fatale avec les monstres. La gestuelle de l'actrice change avec son équipement: ses mouvements deviennent méthodiques et contrôlés, à la fois ralentis et assurés par le poids de ses armes. Elle se comporte comme les marines avant leur débandade, et cette transformation culminera avec l'utilisation du robot monte-charge. Nous assistons donc à un degré supplémentaire de masculinisation du personnage, par 
rapport au premier épisode et par rapport à la plupart des films d'exploitation qui ont précédé ${ }^{23}$. La volonté de plaire au (grand) public féminin états-unien est la première cause de cette évolution. Cependant, transformer Ripley en une combattante insensible risquerait d'aliéner certaines parties du public. La nouvelle vocation maternelle de Ripley a donc son utilité. Son rapport étroit avec Newt fait d'elle une single mother idéale: indépendante, armée, mais sensible et généreuse, elle devient un personnage positif pour l'immense majorité des spectateurs.

Vasquez, la chicana armée d'une mitrailleuse, sert alors à bien montrer au public ce que n'est pas Ripley: une femme-hom$\mathrm{me}^{24}$. Sans être négative ou ridicule, Vasquez est tout de même trop masculine pour jouer le rôle maternel de l'héroïne. Elle prend donc sur elle ce que l'on ne peut attribuer à celle-ci, qui doit rester suffisamment sexuée pour être mère. Mais pourquoi cette nouvelle mère idéale doit-elle ressembler à un soldat?

Au cours des trois dernières décennies, l'Occident, mais surtout les États-Unis, ont vu rapidement évoluer la perception du corps. De nouvelles pratiques culturelles, telles que le body building ou les régimes alimentaires mis au point "scientifiquement ", cherchent à atteindre un but nouveau: contrôler plus efficacement notre corps ${ }^{25}$. Mais le changement le plus important accompagnant cette évolution récente est la participation complète des femmes états-uniennes à ces pratiques. Désormais présentes de manière active à tous les niveaux de la société, les femmes ont dû se libérer de la plupart des traits que notre culture liait à la féminité il y a encore quelques décennies. Leur perception du corps en a été nécessairement modifiée.

Voilà, à mon avis, la principale cause de l'apparition et de la popularité de Ripley: l'idéal corporel du public middle class a changé. Aliens était le film idéal pour marquer cette évolution, ce qui le rend très important à mon sens et qui est la raison de son succès. Alien 3 confirmera que ce corps maîtrisé est la condition sine qua non de notre identification.

\section{Alien 3, histoire d'une fille perdue: "The bitch is back"}

Alien 3 se situe dans la continuité immédiate du deuxième épisode. À bord du navire ramenant Ripley, Newt et Hicks vers 
la Terre, un incendie déclenché par une larve provoque l'éjection du module d'hibernation. Le véhicule s'écrase sur Fury 161, une planète abandonnée par la Compagnie qui sert maintenant de prison à un petit groupe de criminels sexuels violents. Ici, la seule source de réconfort réside dans une vague sensation de communauté virile existant entre les prisonniers. L'ensemble du film montre que le petit groupe a été exclu de la société et vit ici oublié de tous. Malgré un filmage très publicitaire, l'atmosphère générale du film est particulièrement sinistre et déprimante. Il n'y a jamais aucun espoir pour Ripley ou les détenus et une fin tragique est la seule conclusion possible.

\section{Constats, dissonances et contradictions}

Rendre compte des contradictions qui marquent Alien 3 pourrait constituer un travail aussi important en taille que celuici. Les oppositions extrêmes qui séparent les personnages en sont la matérialisation évidente et montrent combien nous sommes loin des certitudes d'Aliens. Pour ne choisir que l'exemple le plus évident : s'il est vrai que le film dénonce la volonté de contrôle de la Compagnie sur le corps de Ripley, nous ne pouvons ignorer que celle-ci va donner naissance à un monstre dangereux. La misogynie féroce des détenus est implicitement justifiée par cette simple constatation. Les problèmes de classes posés par le film reçoivent une solution tout aussi déconcertante, comme nous le verrons.

Exclusion, pauvreté, sida, drogue, ces images évoquées par le film ne sont pas celles que le public visé recherche lorsqu'il se rend au cinéma. Avec de telles prémisses, Alien 3 ne pouvait que décevoir. Mais malgré la validité de cet argument, il me semble nécessaire de chercher la source finale de cette déception dans les questions soulevées par le discours inconscient du texte. J'essaierai de montrer ici que le malaise, le sentiment d'insatisfaction laissés par le film, viennent de la manière dont il répond à ces questions.

Le sous-titre de l'affiche états-unienne donne le ton: sous une photo de Sigourney Weaver est inscrite la phrase "The Bitch is back». Cette phrase volontairement ambiguë indique combien le film est le prolongement des précédents: l'incertitude autour 
du personnage de Ripley demeure entière et les mêmes interrogations restent à résoudre.

\section{"Boys will be boys"}

Après la chute du module d'hibernation, nous découvrons la petite communauté qui habite Fury 161. Leur gardien donne le ton: cet événement ne doit pas troubler l'ordre de la prison et cette femme sera gardée soigneusement à l'écart. Personne ne doit s'inquiéter, il a appelé une "équipe de secours" qui devrait arriver rapidement et les sauver de cette intrusion ${ }^{26}$. Nous comprenons que cette arrivée trouble une harmonie difficilement atteinte et que chacun craint de perdre.

Trois personnages sont mis en valeur par le film et ce sont leurs relations avec Ripley qui en éclairent le sens. Du côté des prisonniers, leur chef spirituel, Dillon, sort du lot. Éloquent et charismatique, il est encore plus endurci que ses compagnons et c'est lui qui leur dicte leur conduite. La religion qu'il professe semble basée sur un rejet total du désir et des pulsions incontrôlés, et en particulier lorsqu'ils sont dirigés vers une femme. Il n'hésite d'ailleurs pas à exprimer un rejet méprisant pour Ripley dès qu'il le peut. La séquence où Ripley lui demande de la tuer met bien en valeur cette attitude: dès qu'ils en auront fini avec la première créature, il la tuera sans hésiter. Face à la femme ou au monstre, pas de sentiment.

Face à Dillon se trouve le surintendant de la prison. Manifestement, les deux hommes se détestent, tout les sépare et il est clair qu'ils n'hésiteraient pas à s'entre-tuer. Cependant, un point les met d'accord: la seule femme présente est de trop. Et la tentative de viol que subit Ripley leur donne bientôt raison. Lorsque Dillon arrive sur les lieux, il frappe violemment ses agresseurs avec une barre de fer. "J'ai à faire un peu de rééducation avec ces frères " explique-t-il à Ripley. Contre le désir, Dillon n’a qu'une réponse, les coups. Et il est clair qu'il ne fait pas cela pour la sauver, mais bien pour ramener ses compagnons dans le droit chemin, pour les guérir de leurs pulsions.

Le bras droit du chef de la prison, surnommé " 85 , pour son quotient intellectuel ", est aussi un membre à part du groupe. Peu courageux, il préfere attendre que l'équipe de secours 
envoyée par la Compagnie arrive et tue le monstre. Même s'il comprend finalement le but véritable de la Compagnie et tente de l'arrêter, le film ne le dépeint pas comme un personnage positif, il est au mieux pitoyable, et au pire, veule et égoïste.

85 explique par deux fois qu'il ne veut rien tenter, car il va bientôt être relevé et qu'il veut revoir sa "femme et son gosse". Lorsque Ripley essaie de renvoyer la navette de secours pour que la Compagnie n'apprenne pas l'existence de la Reine, il l'en empêche pour les mêmes raisons. L'origine de cette lâcheté est intéressante: une femme et un enfant. Comme lui dit Dillon, "You're not one of us, you're a fucking [en effet] Company man!» Il est clair que, contrairement à eux, 85 a été suffisamment proche d'une femme pour avoir un enfant et c'est bien cette caractéristique que le film met en évidence pour l'écarter du groupe. Après tout, nous savons que la Compagnie ne veut pas tuer le monstre et que la lâcheté de cet homme met en danger l'humanité entière. Décidément, il ne faut pas faire du sentiment avec ce genre de créature.

Il existe tout de même un personnage qui se rapproche de Ripley, le médecin Clemens. Éduqué et hétérosexuel, il n’appartient ni à leur ordre ni à leur classe sociale inférieure. Ripley n'hésite pas à exprimer son désir pour lui, mais leurs rapports charnels n'apparaissent pas à l'écran sauf pour les deux piqûres de Clemens. Fétichisés à l'extrême par de très gros plans, ces moments sont les seuls érotiques du film. De façon très artificielle, Clemens reste incroyablement froid. Juste après qu'ils aient fait l'amour, il s'empresse de se lever et de s'habiller, alors que Ripley voudrait prolonger ce bref contact. Après la seconde piqûre, il accepte de lui parler de son passé. Le monstre apparaît alors derrière lui, le soulève et le tue en lui perçant le crâne.

\section{Le monstre, menace extérieure}

Se cachant dans les sous-sols de la prison, celui-ci reste aussi dangereusement bisexuel que son prédécesseur d'Alien. Alien 3, connaissant les vœux de son public et disposant de beaucoup d'argent, multiplie ses apparitions. Répété quatre fois, le processus est toujours identique. Un homme lève les yeux et le voit. Le contrechamp montre la tête ou la gueule baveuse et dentée, 
menace féminine s'il en fut. Le plan suivant montre l'homme paralysé par la terreur, comme l'étaient les victimes de Méduse. Puis, changeant brusquement d'identité sexuelle, le monstre le soulève en le saisissant par la tête et la lui perce de sa deuxième bouche, faisant invariablement jaillir un flot de sang.

Même chose pour son environnement, reconstruction fantasmée du corps maternel dévorant typique du film d'horreur, et de cette trilogie en particulier. Lieux clos sombres et humides, sans espace ni perspective, longs couloirs étroits, tout y est fait pour perdre personnages et spectateurs. Le film est d'ailleurs presque entièrement constitué de gros plans montés très rapidement qui rompent sans cesse la continuité spatiale et achèvent de nous désorienter.

Montée en parallèle avec la crémation des restes de Newt et Hicks, la naissance du monstre précise bien le sens global du film. Le sermon que prononce Dillon pour la cérémonie prend alors un double sens particulièrement sinistre. Ses paroles, avec leurs lourdes connotations religieuses, sont une célébration du miracle de la naissance, de la vie biologique qui parvient toujours à se reproduire et à survivre. Le montage parallèle dénonce cet amour de la vie: c'est l'innommable qui est en train de prendre forme dans les sous-sols de la prison. Pour Alien 3, la vie organique est profondément cauchemardesque, source d'horreur et de destruction. C'est aussi la naissance, symbole du pouvoir reproducteur féminin, qui est ici l'objet de dégoût, de répulsion du film.

À un deuxième niveau, la naissance du monstre est mise en parallèle avec la mort de Newt et Hicks: la famille meurt, la femme reste seule, le monstre naît. Nous sommes alors bien loin d'Aliens. Cette relation étroite entre féminité et monstruosité est renforcée par le saignement de Ripley. Provoqué par la Reine qui grandit en elle ou par toute autre cause, cet écoulement fait écho aux flots de sang qui entourent le "nouveau-né " et, en fait, à toute sa biologie qui est en train de la rattraper. Son corps la lie irrémédiablement avec ce qui se passe en dessous, comme la découverte de la Reine le confirmera. 


\section{Le corps féminin comme véritable menace}

Ripley va devoir reconnaître elle-même cette situation un peu plus tard. Juste après avoir tué Clemens, la créature se jette vers elle et l'accule à un mur. Ripley glisse à terre, terrifiée. La bête approche alors sa gueule tout près de son visage. C'est le clou du film, l'image la plus frappante de la bande-annonce, celle qui a poussé un grand nombre de personnes à venir le voir. Il va la tuer, mais il s'arrête et semble comprendre quelque chose qui le fait changer d'avis. Il est évident que cette scène trouve une partie de son pouvoir horrifiant dans le crâne et la bouche phalliques menaçant Ripley. Mais ce qui frappe, c'est qu'il ne la tue pas, il la reconnaît. Cette promiscuité sinistre parce que inexplicable nous laisse dans le doute, et l'horreur véritable vient de cette incertitude.

Le film ne nous laisse d'ailleurs pas longtemps douter ainsi, puisque Ripley découvre une larve dans sa poitrine. Bien sûr, il ne s'agit pas de n'importe quelle larve, c'est celle d'une reine, un monstre dont le pouvoir reproducteur peut générer "des milliers de ses semblables ". Décidée à mourir, elle part seule à la rencontre du monstre. Lorsque 85 lui demande comment elle espère le trouver, elle répond: "Pas besoin de chercher, je sais où il se cache." Arrivée dans ce qu'elle croit être sa tanière, Ripley se met à lui parler: "Je fais partie de la famille", "Tu es dans ma vie depuis si longtemps que je ne me souviens plus de rien d'autre». Ces deux phrases donnent une idée du lien établi par le film entre femme et monstre. Lien d'ailleurs confirmé par le montage, qui associe systématiquement les meurtres du monstre avec Ripley.

\section{Chute de classe, corps et monstruosité}

Au cours du deuxième épisode, la distinction entre Ripley et les parasites était bien établie. À la fin du film, la famille reconstituée s'échappait saine et sauve de l'antre nauséabonde de la famille matriarcale vivant "en bas».

Le troisième épisode précipite Ripley au ban de la société, là d'où on ne remonte pas: au fond d'une prison perdue au milieu de nulle part. Le personnage principal, membre obligé de la middle class, pénètre ici dans un espace délabré et sordide qui n’a 
jamais été le sien, et hors duquel elle aurait pu avoir une autre chance. Même si les prisonniers sont montrés comme des personnages plutôt positifs, la plupart sont des brutes épaisses avec qui Ripley n'a rien à voir. Cette différence de classe est confirmée par Clemens, que seul son statut social supérieur autorise à approcher l'héroïne.

Comme l'explique Kristeva, la société occidentale parvient toujours à un équilibre dans sa gestion des rapports entre hommes et femmes, mais cet équilibre n'existe que si les institutions sociales contrôlent efficacement le féminin. Or, Alien 3, conçu par la middle class, pour la middle class, associe pauvreté et pulsions incontrôlées. La chute de classe éloigne la femme des institutions qui ordonnent la société et les relations entres les sexes. Et c'est dans cette univers dégradé que femme et monstruosité peuvent se rencontrer. Ce lien entre pauvreté et régression vers le monstrueux est important, car il montre que la femme est perçue comme intrinsèquement dangereuse par notre culture.

\section{Pourquoi Ripley meurt-elle?}

Quelle est la conséquence de cette déchéance? Parallèlement à sa masculinisation extrême, Ripley adopte par deux fois un rôle typiquement féminin. Et à deux reprises, cela provoque le désordre et la destruction. Elle libère d'abord involontairement les pulsions des prisonniers qui essaient de la violer. C'est la première fois qu'elle est ainsi menacée. Auparavant, même à la fin du premier épisode, Ripley courait un danger réel, mais qui n'était pas lié au caractère biologique féminin. D'ailleurs, à la fin de la séquence, elle frappe violemment un de ses agresseurs, comme pour bien se convaincre, et nous convaincre, qu'elle n'est pas une faible femme.

La sexualité de Ripley apparaît, pour la première fois de la série, avec Clemens. Contrairement aux deux autres épisodes, Ripley fait l'amour avec un homme et perd ainsi la caractéristique principale de la final girl: l'absence de sexualité. Nous avons vu les conséquences de ce changement: Ripley ne peut plus gagner. Mais que voulait dire "gagner» au début du film, dès le début, en fait, de la trilogie? 


\section{Ripley et Arnold, même combat?}

Il semble donc que la véritable source d'insatisfaction ressentie face au film tienne dans son incapacité à résoudre les contradictions qu'il a exposées. Les problèmes à résoudre sont simples, nous les avons évoqués: qu'y a-t-il à l'intérieur de Ripley? Estelle, en tant que femme, vraiment liée au monstrueux? Va-t-elle réussir à s'en démarquer définitivement?

Alien 3 perd sa cohérence, car il aborde des problèmes que la culture états-unienne est elle-même incapable de résoudre. Les bouleversements qui agitent le société perturbent, parfois, le discours des films qui abordent ces questions et les empêchent d'y donner une seule réponse. Plus que tout autre aspect de cette culture, le film reflète sa confusion actuelle par rapport à ces thèmes.

La seule réponse claire est l'incinération de la femme ${ }^{27}$. Les deux premiers épisodes tuaient, de manières différentes, le monstre. Alien 3 a changé d'avis sur sa véritable origine, mais la supprime aussi définitivement. Mais cette résolution n'est pas heureuse pour le spectateur, non seulement parce qu'elle tue l'héroïne, mais surtout parce qu'elle nous laisse face à l'altérité de la femme. Là où ses deux prédécesseurs arrivaient à des conclusions rassurantes, le film arrive à une impasse totale dans sa représentation de la femme et de ses rapports avec l'homme.

La fin d'Alien 3 ressemble de façon remarquable à celle de Terminator 2. La similitude remonte même beaucoup plus loin. Les deux personnages se sont forgés une réputation de soldats efficaces en utilisant une technologie guerrière dont le principal effet est d'empêcher tout contact physique.

Mais la ressemblance s'arrête au moment où Ripley tombe dans le métal en fusion. Là où Arnold Schwarzenegger levait triomphalement un pouce rigide en direction de sa descendance masculine, Ripley ne peut que serrer contre sa poitrine une petite abomination qui, heureusement pour l'humanité entière, sera consummée avec elle.

Le cyborg, façonné par des machines et interprété par Monsieur Univers, concrétisait sa victoire sur l'homosexuel protéiforme en subissant volontairement le sort qu'il venait de lui infliger. Comme il l'explique au jeune garçon qui, lui, souffre de 
le quitter, Arnold "ne peut pas pleurer». Enfin totalement libéré de ce qui aurait pu mettre en danger son invulnérabilité, les sensations ou les sentiments, le héros pouvait cesser, non de vivre, juste d'exister.

Ripley est encore désespérement vivante lorsqu'elle tombe, tellement vivante qu'elle a encore le temps de donner naissance à un être vivant, lui-même tellement vivant qu'il pourrait donner naissance à des "milliers d'autres". Face à l'immobilisation finale de l'homme-machine, sa mort fait désordre. Le Terminator triomphait face aux corps et à la corporation démonisée, Ripley perd face à son corps et à la Compagnie ${ }^{28}$. Faut-il en conclure que les deux combattants n'ont pas eu la même détermination?

Peut-être: Ripley voulait vivre, retrouver une famille, faire l'amour, relations basées sur le contact avec d'autres êtres humains. Sa perte a bien sûr été causée par son corps de femme, mais aussi par cette volonté de ne pas renoncer à ses émotions. Elle l'avait pourtant compris dans Aliens: contre elles, il ne faut pas faire de sentiment.

Les deux séries parviennent donc à des conclusions différentes en partant de la même conviction: ce que notre culture définit comme le féminin est une force hostile, dangereuse, car capable de détruire notre identité. Le film fantastique hollywoodien est particulièrement intéressant car, en nous forçant à entrer dans la peau de ses personnages, il nous apprend où, dans la culture états-unienne, se situe le siège de cette identité. En s'acharnant sur le corps de ses victimes, c'est-à-dire en le plaçant comme centre de notre regard puis en le détruisant, ce cinéma lie directement cette identité à notre intégrité corporelle.

Le sort de Ripley montre ce qui guette en fait tous les personnage de ces films, quel que soit leur sexe: comme le Terminator essaie de le faire, elle cherche continuellement à échapper à ce qu'il y a de féminin en elle. Mais elle part avec un lourd handicap. Toujours trop proche, par son corps de femme, de l'Inconnu, du monstrueux, elle tend désespérément vers une masculinisation qui la sauverait de sa propre biologie. Dans Alien, elle y parvenait en fuyant le corps maternel difforme et en expulsant son rejeton androgyne. Aliens rejetait toute trace de féminité dangereuse hors de Ripley et rassemblait ces traits 
négatifs dans un "utérus carnivore" aux proportions démesurées. Alien 3 renonce finalement à séparer femme et féminin et nous montre les terribles conséquences de cette réunion.

Si nous comprenons que le spectateur s'identifie au personnage principal, c'est-à-dire à celui qui correspond à notre idéal corporel, le danger qui le guette est toujours le même. Tous ces personnages cherchent à devenir invulnérables pour ne pas être absorbés, pénétrés, ouverts, pour ne pas couler et se répandre, c'est-à-dire pour ne pas être réduits à l'expression essentielle de la féminité d'après la culture occidentale: le magma informe qui nous a donné naissance, ce qu'il y a "à l'intérieur». Une femme aussi peut se séparer du "féminin", non seulement elle le peut, mais elle le doit. Aussi triste que cela puisse paraître, celle qui n'y parvient pas sera... brûlée.

La trilogie Alien permet de commencer à comprendre une donnée fondamentale du système de représentation hollywoodien. L'analyse du personnage de Ripley, de l'évolution de son rôle et de son corps, montre que ce cinéma lie inextricablement représentation et statut narratif. Plus que tout autre élément, c'est bien le corps de la femme qui la positionne à l'intérieur de l'histoire, qui dicte son comportement et son destin. Maîtrisé, il lui permettra de garder un rôle actif et de vaincre tous les obstacles. Mais qu'apparaissent la chair et ses faiblesses, et la femme sera, encore une fois, déchue, entraînant avec elle ceux qui furent assez fous pour établir un tel contact.

\section{Université Paris III-Sorbonne Nouvelle}

\section{NOTES}

1 Theweleit analyse notamment les livres écrits par les anciens membres des Freikorps.

2 Un long et massif cylindre, placé dans son axe et pointé vers le ciel, domine le pilote.

3 Cette image est déjà présente dans les orifices par lesquels les explorateurs pénètrent dans l'épave.

4 Les parasites sont dotés d'un orifice lui aussi quasi vaginal et possèdent huit pattes griffues qui en font de véritables "vagins dentés", saisissant leurs victimes pour ne les lécher qu'après les avoir inséminées. Mais cette insémination est aussi un viol. Nous reviendrons sur ce caractère androgyne. 
5 La clarté intense et la propreté qui règnent dans cette salle l'opposent totalement à l'épave d'où jaillira la mort. Ici, chacun est clairement séparé des autres par un cocon disposant d'une grande paroi vitrée. Tout se fait avec précision, dans le calme et une intense impression de sécurité se dégage de la séquence.

6 N'oublions pas que Mother la protège constamment. En dissimulant l'origine de la tache sombre qui remplit la cage thoracique de Kane, lorsque Ash empêche Parker d'attaquer la chose après sa "naissance", et lorsqu'il parvient à l'alerter avant que l'équipage ne puisse l'enfermer dans un sas.

7 En 1978, Ripley est en effet la première femme forte à faire son entrée dans le cinéma mainstream. Dans son livre, Clover analyse longuement le personnage de la final girl, en se basant sur des séries telles que Friday the Thirteenth ou Halloween. Ce personnage, intelligent et déterminé, apparu depuis le milieu des années soixante-dix dans le cinéma d'exploitation, parvient, au prix d'une lutte acharnée, à survivre et à triompher in extremis du mal. Se retrouvant invariablement seule, elle se transforme et lutte pied à pied, faisant preuve de toutes les qualités masculines nécessaires à la victoire. Le public est alors de son côté, face à son ennemi, et triomphe avec elle. Alien et ses suites ne feront que récupérer à leur profit une tendance dont le succès est largement éprouvé.

8 Ici et pour le reste de ce travail, le terme "producteur" désigne l'ensemble des individus ayant présidé à la création et à la vente d'un film. Nous supposons, sauf exception, que leur principal objectif était alors d'assurer son succès.

9 Clover écrit en page cinq de son livre Men, Women and Chainsaws (Londres: BFI-Princeton University Press, 1992): "[Il est possible] que le spectateur masculin soit tout à fait prêt à s'identifier non seulement aux personnages féminins, mais aussi aux personnages féminins des films d'horreur, plongés dans la peur et la douleur. Cette identification, la négation de cette identification et les implications plus générales de ces deux éléments sont le sujet de ce livre. " Ma traduction.

10 Certains spectateurs, hommes ou femmes, peuvent alors prendre plaisir à voir le monstre tuer ses victimes, notamment parce que celles-ci semblent tout faire pour précipiter leur propre trépas. Ces différents types d'identification soulignent la fluidité du rapport entre le public et le film. C'est d'ailleurs cette fluidité qui invalide le plus certainement les conclusions de Mulvey.

11 Les systèmes narratifs proposés par des séries telles que celles qu'analyse Clover sont simples et répétitifs. À tel point que tout spectateur ayant vu ne serait-ce qu'un épisode connaît parfaitement la direction que prend n'importe quel autre film de ce type. Mais cela n'enlève pas au public le plaisir de se faire peur en toute connaissance de cause. Dans le cas d'Alien, dont la facture générale semble plus subtile, ces certitudes sont plus fragiles, ce qui créera une peur plus brute, et assurera le succès du film.

12 Et le cinéma hollywoodien en général (Carol J. Clover, Men, Women and Chainsaws, Londres: BFI-Princeton University Press, 1992, p. 63). Le personnage de Lambert, écho des innombrables jeunes femmes massacrées par les films d'exploitation, continue, elle, à se comporter en femme typique du film d'horreur. Toujours terrorisée, elle finit par paniquer et propose de quitter le Nostromo sans essayer de secourir les disparus. Le film montre que son comportement ne peut que précipiter sa mort. Lorsqu'elle se retrouve face à la créature, elle reste paralysée de terreur. C'est d'ailleurs face à elle que le monstre devient "masculin ".

13 Dès le début du film, la final girl se différencie déjà des autres. Ses deux principales caractéristiques sont la clairvoyance (elle comprend le danger qui menace le groupe) et un désintérêt total pour le sexe souvent lié à une apparence plutôt androgyne. Cette transformation finale accentue encore la différence entre elle et ses compagnons. 
14 Le choix de Sigourney Weaver, alors quasi inconnue à Hollywood, est déjà un pas dans ce sens. Dès Alien, son visage carré et son allure générale, mêlant sévérité et androgynie, la prédisposait au rôle de final girl.

15 Réalisateur de The Terminator (1984) et de Terminator 2 (1992) qui expriment différemment des angoisses très semblables. Voir Sylvestre Meininger., "Terminator 2, technologie et identité sexuelle", Cinémas, vol. 5, n³ (1995), p. 131-150. Par ailleurs, sa réalisation The Abyss (1990) construit un Autre bienfaisant, mais reste tout aussi conservateur dans sa reconstitution implacable du couple patriarcal.

16 Rappelons à ce titre que, aussi bien dans le film que dans les divers jeux et bandes dessinées qu'il a inspirés, les soldats sont appelés Colonial Marines.

17 Burk essaie en effet de faire inséminer Newt et Ripley afin de ramener des spécimen sur Terre pour son propre profit.

18 Cette entente tacite entre les deux mères renvoie directement au problème que le film doit résoudre: séparer le féminin supportable du féminin abject. La violence utilisée pour cette séparation montre d'ailleurs à quel point le film craint un tel rapprochement.

19 Ce personnage est interprété par Michael Biehn, acteur fétiche de James Cameron, qui incarnait déjà Reese dans The Terminator. Il joue ici un rôle semblable d'homme idéal, c'est-à-dire de guerrier compétent mais capable de sentiments. C'est lui qui apprend à Ripley à utiliser une arme et à se défendre. Comme dans The Terminator, il est montré comme entièrement positif et est évacué vers la fin pour laisser place à l'héroïne.

20 Voir bien sûr Fatal Attraction (A. Lyne, 1986) ou, et plus récemment, The Hand That Rocks the Craddle ou Single White Female...

21 Voir à ce sujet Barabara Ehrenreich, Fear of Falling. The Inner Life of the Middle Class (New York: HarperCollins, 1990).

22 Les robots humanisés et l'homme-singe de Star Wars (G. Lucas, 1977) ont un comportement assez semblable et servent eux aussi à mettre en valeur le statut social supérieur des humains-héros, deux termes complaisamment confondus par la trilogie.

23 Précisons qu'à présent, et notamment depuis Aliens, les final girls ressemblent très souvent à Ripley.

24 Tout au long du film, Vasquez est même caractérisée comme "plus " masculine que ses compagnons. Par exemple, lorsqu'un soldat lui demande: "Vasquez, on ne t'a jamais prise pour un homme?", elle répond: "Non, et toi?" Elle est plus courageuse, plus forte, compétente dans tous les domaines et elle meurt héroïquement. D’après mon expérience personnelle, elle est le personnage secondaire préféré du public.

25 Bien sûr, la détérioration des conditions de vie dans les pays industrialisés et le poids de plus en plus important de la compétition entre les individus sont aussi à l'origine de cette volonté de contrôle. Mais nous devons aussi la considérer comme l'aboutissement logique de l'évolution de la perception occidentale du corps. Évolution que Theweleit fait remonter à la fin du Moyen Âge, lorsque l'homme occidental commença à étendre son contrôle sur des territoires de plus en plus importants.

26. Le dialogue laisse en effet planer le doute sur le but de cette "équipe de secours " : sauver Ripley des prisonniers ou l'inverse?

27 S'il est vrai que Sigourney Weaver souhaitait en finir avec le personnage de Ripley, sa mort conserve toute sa signification dans le cadre de mon analyse. En effet, même si cet événement de la narration a une origine en partie extradiégétique, le résultat à l'écran est le même: Ripley meurt pour sauver l'humanité.

28 Autre différence, Ripley a un nom, une identité, preuve supplémentaire de son humanité, et donc de sa fragilité par rapport à l'impénétrabilité inhumaine de l'homme-machine. C'est aussi pour cela que nous sommes émus par sa mort. 


\section{OUVRAGES CITÉS}

Bundtzen, Linda K. "Monstruous Mothers, Medusa, Grendel and Now Aliens». Film Quarterly, vol. XL, n³ (1987) p 11-17.

Clover, Carol J. Men, Women and Chainsaws. Londres: BFI-Princeton University Press, 1992.

Ehrenreich, Barabara. Fear of Falling. The Inner Life of the Middle Class. New York: HarperCollins, 1990.

Klein, Mélanie. L'Amour et la haine. Paris: Payot, 1975.

Klein, Mélanie. Envy and Gratitude, and other Works. London: Hogarth Press, 1975.

Kristeva, Julia. Pouvoirs de l'horreur. Paris: Seuil, 1980.

Mahler, Margareth. La Naissance psychologique de l'être humain. Paris: Payot, 1980.

Mahler, Margareth. On Human Symbiosis and the Vicissitudes of Individuation. New York: International Universities Press, 1968.

Mulvey, Laura. "Visual Pleasure and Narrative Cinema». Screen, vol. 16, n" 3, (1975) p. 8-18.

Theweleit, Klaus, Male Fantasies, vol. I et II. Minneapolis: University of Minnesota Press, 1987, 1988. 